\title{
Verification of a treatment planning system using an in-house designedtrunk phantom
}

\author{
AkpochaforMO,AwedaMA,Omojola AD, Adeneye SO'Iloputaife C \\ Department of Radiation Biology, Radiotherapy, Radiodiagnosis and Radiography \\ College of Medicine, Lagos University Teaching Hospital, Lagos, Nigeria.
}

\begin{abstract}
This study is aimed at verifying the dosimetric performance of a treatment planning system (TPS) and to as well compare the accuracy of the measured absorbed dose of the solid water phantom against the inhouse phantom. In this study, a phantom for use in radiotherapy treatment planning of human trunk anatomical region has been designed with six hollows for inserting materials mimicking different biological tissues and the ionization chamber. For the trunk, pure Glycerolwas used for Muscle, 75\% to 25\% Glycerol-Water was used for liver, Carboxyl-Methyl-Cellulose (CMC)was used for Lungs, 50\% to 50\% Glycerol-Water was used for Adipose, Sodium Hypochlorite (soda bleach) for Bone and SodiumLaureth Sulphate (Texapon) was used for Kidney. The phantom was scanned with Hi-Speed CT-scannerand images were transferred to a Precise PLAN Treatment Planning System where appropriate beams were applied and verified before it was transferred to the Elekta-Precise Clinical Linear Accelerator.Measurements of the Monitor Units (MU) were conducted using 6 $\mathrm{MeV}$ photon beams from the Elekta-Precise Clinical Linear Accelerator with iso-centric set up and the corresponding doses were calculated. The test of the phantom was done using an Irregular Field Algorithm (Clarkson Integration). The maximum standard deviation with large field size of $22 \times 24 \mathrm{~cm}^{2}$ for all sixinhomogeneous inserts and bone only inhomogeneous inserts were $-3.39 \%$ and $2.93 \%$ respectively. And maximum standard deviation with small field size of $5 \times 5 \mathrm{~cm}^{2}$ was $-3.16 \%$. Also, the percentage deviation for the solid water phantom when compared with the in-house phantom with SSD of $85 \mathrm{~cm}$ for both set-ups was $-2.09 \%$. Theseresultsshow that irrespective of the field sizes and tissue equivalent materials, Irregular Field Algorithm compensates for inhomogeneity.
\end{abstract}

Key words: Phantom, Treatment Planning System (TPS), Irregular Field Algorithm, Clarkson Integration, Computed Tomography.

\section{Introduction}

Radiotherapy aims to cure, or locally control disease, while concurrently minimizing complications in normal tissue. The International Commission on Radiation Units and Measurements (ICRU) has recommended that radiation dose must be delivered to within $\pm 5 \%$ of the prescribed dose[2, 10,15$]$. For a centre using a conventional treatment technique, which is based primarily on measured data[14], there is the need to verify the algorithm in use because quality assurance program ensures that all the components of the treatment facilities used in radiotherapy must be properly checked for accuracy and consistency and that all radiation generating facilities are functioning according to manufacturer's specification. Several technique of carrying out the quality assurance of TPS has been proposed by various authors $[7,8,16,17,18,19]$.Likewise, the reduction of errors and uncertaintiesin the dose calculation plays an important role in thesuccess of a treatment procedure $[6,9,11$, 18].The performance and quality of any Treatment Planning System (TPS) is dependent on the type of algorithm used. An algorithm is defined as sequence of instructions that operate on a set of input data, transforming that information into a set of output result that are of interest to the user[3]. Treatment planning requiresthe ability to calculate dose to any arbitrary point, within the patient, for any given beam orientation. In this study, the Irregular Field Algorithmwas used.Irregular Field Algorithm requires the separation of the dose into primary and scatter components. The concept of thisdosimetry of irregular fields using TMRs and SMRs is analogous to the method using TARs and SARs[14].The magnitude of the dose from scattered radiation at some given point can be quantified using the Scatter-Air or Scatter-maximum Ratios (SARs, SMRs). Equation 1 explains this Irregular Field Algorithm which is based on Clarkson Integration.

In Clarkson Integration, the dose is calculated at a point $(x, y)$ in a plane at depth $d$ as the sum of primary and scatter dose:

$D(x, y, d)=\Phi(x, y)\left[\operatorname{TAR}\left(0, d_{\text {eff }}\right)+\operatorname{SAR}\left(x, y, d_{\text {eff }}\right)\right]$

Where:

$T A R=$ Tissue-air ratio

$S A R=$ Scatter-air ratio

$d_{\text {eff }}=$ Radiological depth 


\section{Materials And Methods}

The designed in-house phantom was made of Plexiglas of thickness $0.33 \mathrm{~mm}$ having a density $1.16 \mathrm{~g} /$ cm3[14]. A plastic based hardener (all-plast) was used for holding one slab to another to form a cube. The Plexiglas used was purchased from a local plastic shop of dimension 4 by 8 feet, a part of which was cut using a plastic cutter into six slabs each of dimension $30 \times 30 \mathrm{~cm}$. Seven holes were drilled on one face. Each drilled hole had a diameter of $2.5 \mathrm{~cm}$ gummed together using plastic based hardener called 'allplast'. Before the holes were drilled, the distance from the surface of the designed in-house phantom tothe ionization chamber was $15 \mathrm{~cm}$, while the distance between two diagonal insert were approximately $22 \mathrm{~cm}$. The distances from one insert to the other (horizontally) was $7 \mathrm{~cm}$ and vertically were $18 \mathrm{~cm}$. Also additional drilled hole was made at the top of the designed in-phantom to allow for easy filling of water and evacuation of water from the phantom. After these holes and distance have been marked out, another cylindrical rod made of Plexiglas material of thickness $0.2 \mathrm{~mm}$, length $14.3 \mathrm{~cm}$ and diameter $2.5 \mathrm{~cm}$ were fitted into the seven drilled holes and were held together at the tip by the 'allplast' gum to avoid leakage. A view of this designed in-house phantom is shown in figure 1 . The in-house phantom was loaded with tissue-equivalent material putting into consideration the attenuation coefficients, electron densities and the effective atomic numbers of each chemical constituent as shown in table 1. The in-house phantom was scanned under a Hi-Speed CT-scanner. Slices of images were acquired for six different tissue-equivalent materials as shown in figure 2. A second scan was conducted for bone only as shown in figure 3. From the acquired CT images, inhomogeneities were determined using Computed Tomography number calculation algorithm. The scanned images were transferred to the precise PLAN Treatment Planning System forbeamapplication as shown in figure 4, 5 and 6. Large field size $\left(22 \times 24 \mathrm{~cm}^{2}\right)$ and small field size $\left(5 \times 5 \mathrm{~cm}^{2}\right)$ were used for this study.

Furthermore, a simple experimental protocol for the verification of the algorithm was performed between the in-house phantom and the solid water phantom with Source to Surface Distance (SSD) of $85 \mathrm{~cm}$. According to this study, the precise PLAN photon beam dose calculation uses an Irregular Field Algorithm based on previously published methods $[5,12,13]$ configured to give $1.0 \mathrm{~Gy}$ at the iso-centre. The optimal plans were then used with the pre-calibrated Elekta-Precise clinical linear accelerator for measurements.

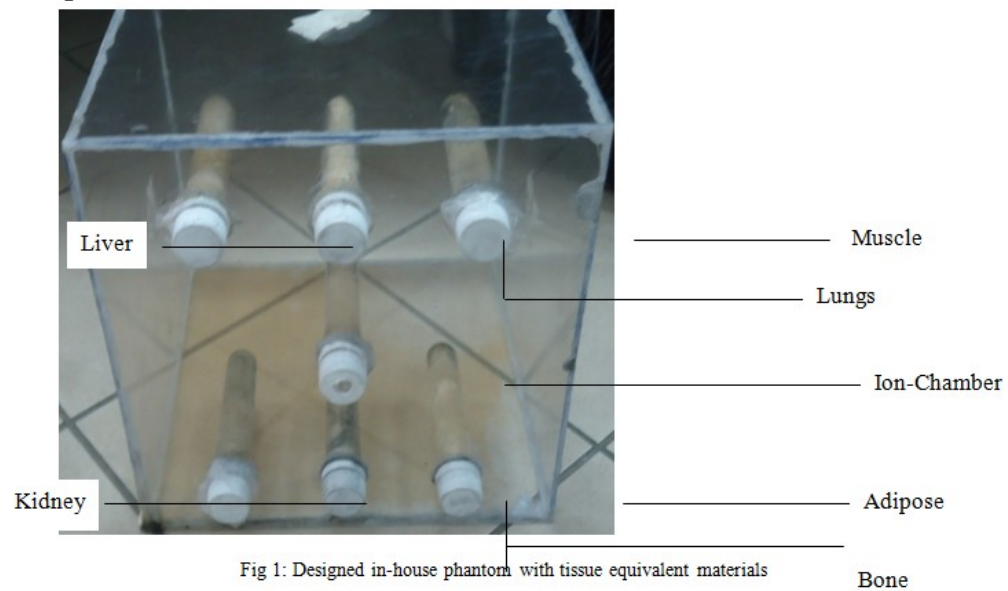

\begin{tabular}{|l|l|}
\hline TISSUE EQUIVALENT MATERIAL & ELEMENTAL COMPOSITIONS \\
\hline Liver & $\begin{array}{l}75 \% \text { of Glycerol } \\
25 \% \text { of water }\end{array}$ \\
\hline Lungs & $100 \%$ of Carboxyl Methyl Cellulose (CMC) \\
\hline Muscle & $100 \%$ of Glycerol \\
\hline Adipose & $\begin{array}{l}50 \% \text { of Glycerol } \\
50 \% \text { of water }\end{array}$ \\
\hline Bone & $100 \%$ Sodium Hypochlorite (soda bleach) \\
\hline & \\
\hline Kidney & $100 \%$ of Sodium LaurethSulfate \\
\hline
\end{tabular}

Table 1: Chemical compositions 

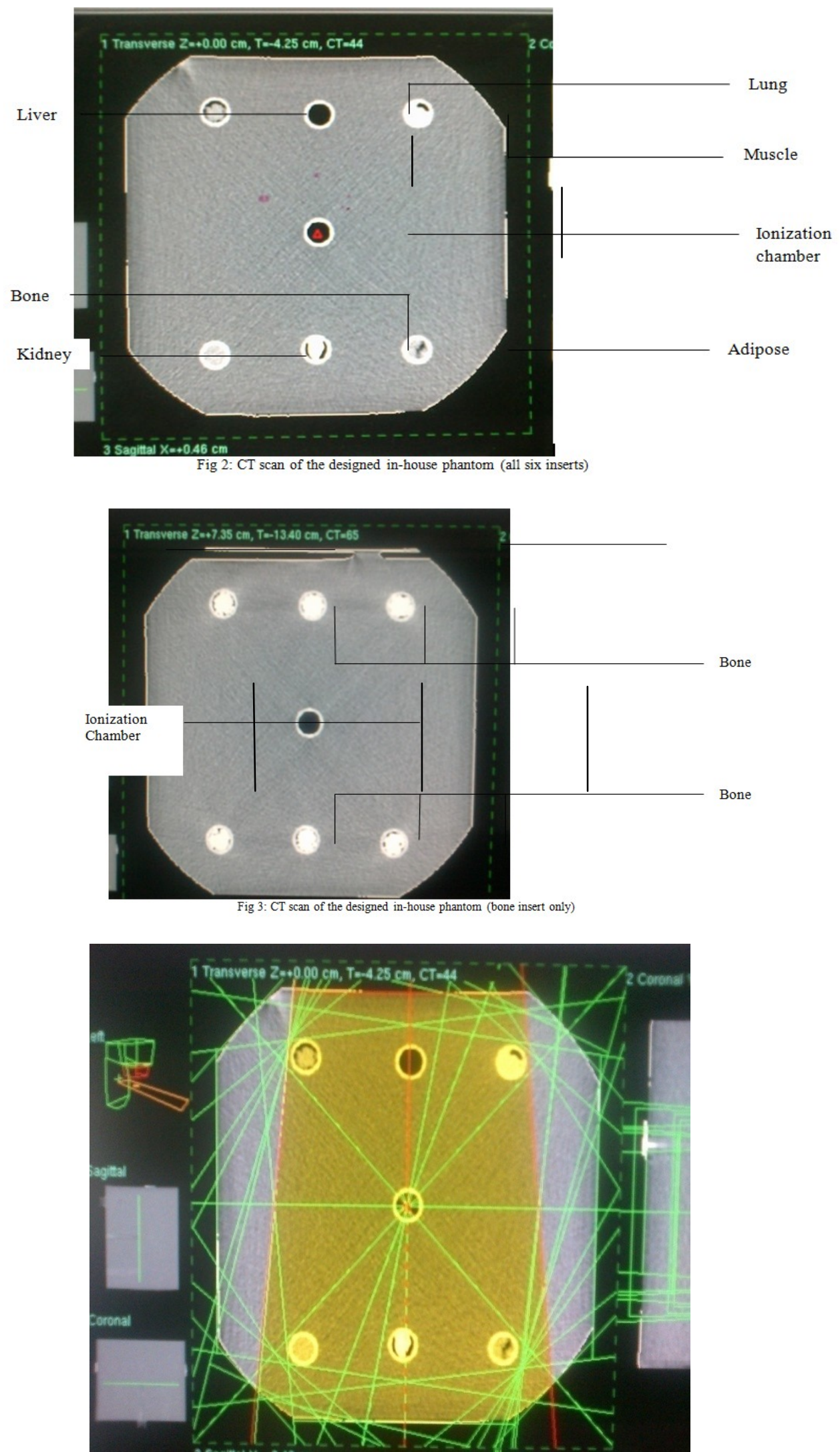

Fig4: Twelve beams with large field size (all six inserts) 


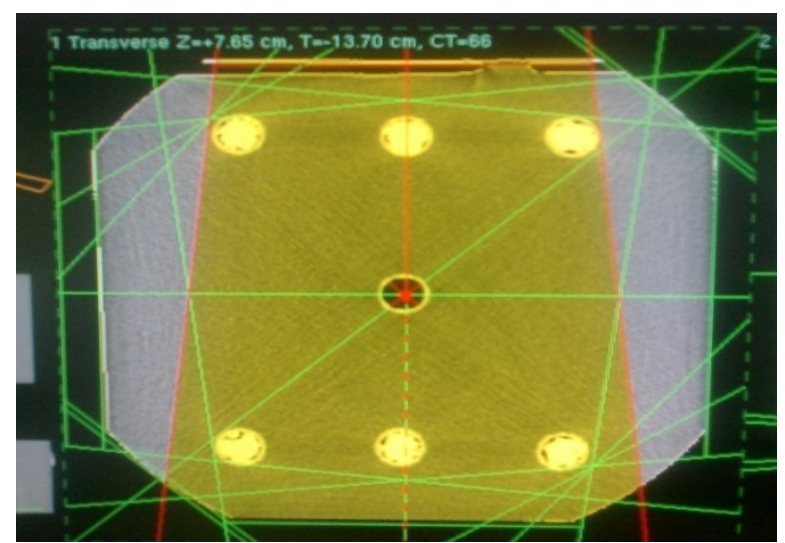

Fig 5: Six beams with large field size (bone insert only)

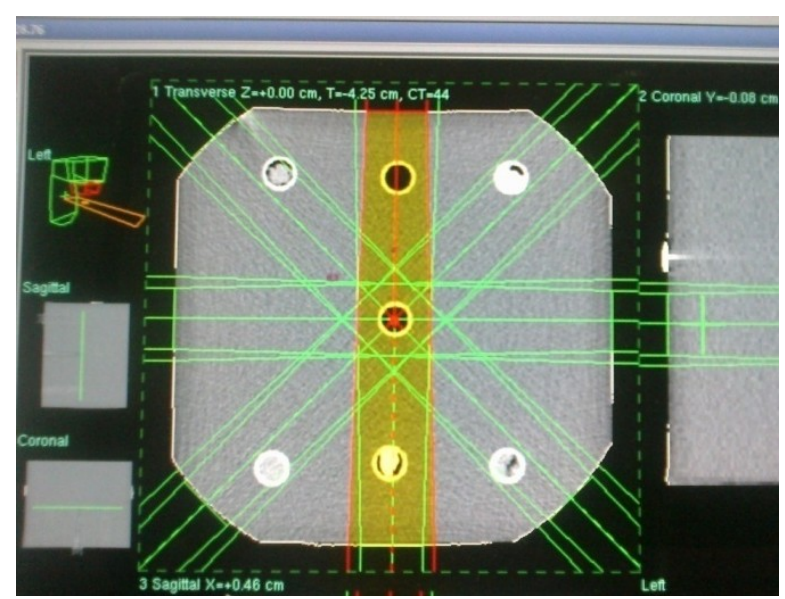

Fig 6: Six beamswith 5 x $5 \mathrm{~cm}^{2}$ Field Size (all six insert)

Measurements were conducted using $6 \mathrm{MeV}$ photon beams from the Elekta-Precise clinical linear accelerator with iso-centric set up. A pre-calibrated NE 2570/1 farmer-type ionization chamber along with its electrometer was used to determine the absorbed dose. Necessary corrections for temperature, pressure, polarization, recombination etc were effected on the ionization chamber response. Five measurements were made in all, four for in-house phantom and one for the solid water phantom. Absorbed dose at reference depth wascalculated as follows [1]:

$D_{W, Q}=M_{Q} \times N_{D, W} \times K_{Q, Q_{0}}$

Where $M_{Q}$ is the electrometer reading (charge) corrected for temperature and pressure, $N_{D, W}$ is the chamber calibration factor and $K_{Q, Q_{0}}$ is the factor which corrects for difference in the response of the dosimeter at the calibration quality $Q$ and at quality $Q_{0}$ of the clinical x-ray beam according to the TRS 398 protocol of the IAEA. Deviation between expected and measured dose was obtained using equation 2

$\%$ Deviation $=\frac{D_{m}-D_{c}}{D_{m}} \times 100$

Where:

$D_{m}=$ Measured dose

$D_{c}=$ Calculated dose

\section{Results}

Table (2a) with field size of $22 \times 24 \mathrm{~cm}^{2}$ for single field-twelve fields with all six inhomogeneous inserts showed that the maximum percentage deviation was $-3.39 \%$. Table (2b) with field size of $22 \times 24 \mathrm{~cm}^{2}$ for six fields with bone only inhomogeneous inserts showed similar maximum percentage deviation which was $2.93 \%$. Table (2c) with field size of $5 \times 5 \mathrm{~cm}^{2}$ for six fields shows a maximum percentage deviation of $3.16 \%$ which is similar to those obtained in table ( $2 a$ and $b)$. Table (2d) shows that the percentage deviation between the solid water phantom and the in-house phantom was $2.09 \%$ 
Verification of a treatment planning system using an in-house designedtrunk phantom

Table 2a: Measured absorbed dose and percentage deviation for all six inhomogeneous inserts with large fields size

\begin{tabular}{|c|c|c|}
\hline & $\begin{array}{c}\text { SOLID WATER } \\
\text { PHANTOM } \\
\text { (Measured absorbed dose) }\end{array}$ & $\begin{array}{l}\text { IN-HOUSE PHANTOM } \\
\text { (Measured absorbed dose) }\end{array}$ \\
\hline & 0.6282 & 0.6076 \\
\hline & 0.6259 & 0.6076 \\
\hline & 0.6282 & 0.6054 \\
\hline & 0.6282 & 0.6054 \\
\hline & 0.6259 & 0.6076 \\
\hline & 0.6282 & 0.6054 \\
\hline STD & 0.0012 & 0.0011 \\
\hline AVG & 0.6274 & 0.6065 \\
\hline & \multicolumn{2}{|c|}{$\% D E V=2.09$} \\
\hline
\end{tabular}

Table 2b: Measured absorbed dose and percentage deviation for bone only inhomogeneous inserts with large field size

\begin{tabular}{|c|c|c|c|c|c|c|}
\hline \multicolumn{7}{|c|}{ TRUNK INHOMOGENEITY $\left(22 \times 24 \mathrm{~cm}^{2}\right.$ FIELD SIZE $)$} \\
\hline FIELDS & \multicolumn{3}{|c|}{ MEASURED ABSORBED DOSE } & STD & AVG & \%DEV \\
\hline 1 & 0.9996 & 0.9996 & 1.0019 & 0.0013 & 1.0004 & 0.4000 \\
\hline 2 & 0.9973 & 0.9973 & 0.9996 & 0.0031 & 0.9981 & -0.1900 \\
\hline 3 & 0.9969 & 0.9954 & 0.9999 & 0.0023 & 0.9974 & -0.2600 \\
\hline 4 & 1.0019 & 1.0041 & 1.0042 & 0.0013 & 1.0034 & 0.3400 \\
\hline 5 & 0.9734 & 0.9813 & 0.9734 & 0.0037 & 0.976 & -2.4000 \\
\hline 6 & 1.0096 & 1.0055 & 1.0096 & 0.0019 & 1.0082 & 0.8200 \\
\hline 7 & 0.9758 & 0.9563 & 0.9758 & 0.0092 & 0.9784 & -2.1600 \\
\hline 8 & 0.9722 & 0.9723 & 0.9722 & 0.0001 & 0.9722 & -2.7800 \\
\hline 9 & 0.9766 & 0.9835 & 0.9858 & 0.0039 & 0.9820 & -1.8000 \\
\hline 10 & 0.9676 & 0.9745 & 0.9562 & 0.0075 & 0.9661 & -3.3900 \\
\hline 11 & 0.9769 & 0.9769 & 0.9586 & 0.0086 & 0.9708 & -2.9200 \\
\hline 12 & 0.9836 & 0.9815 & 0.9746 & 0.0038 & 0.9799 & -2.0100 \\
\hline
\end{tabular}

Table 2c: Measured absorbed dose and percentage deviation for all six inhomogeneous inserts with small field size

\begin{tabular}{|c|c|c|c|c|c|c|}
\hline \multicolumn{7}{|c|}{ BONE ONLYINHOMOGENEITY $\left(22 \times 24 \mathbf{c m}^{2}\right.$ FIELD SIZE) } \\
\hline \multirow{2}{*}{ FIELDS } & \multicolumn{3}{|c|}{ MEASURED ABSORBED DOSE } & \multicolumn{2}{c|}{ STD } & AVG \\
\hline 1 & 1.0300 & 1.0300 & 1.0280 & 0.0012 & 1.0293 & 2.930 \\
\hline 2 & 1.0166 & 1.0166 & 1.0189 & 0.0013 & 1.0174 & 1.740 \\
\hline 3 & 1.0234 & 1.0211 & 1.0257 & 0.0046 & 1.0234 & 0.260 \\
\hline 4 & 1.0120 & 1.0166 & 1.0052 & 0.0051 & 1.0113 & 1.130 \\
\hline 5 & 1.0119 & 1.0142 & 1.0120 & 0.0013 & 1.0127 & 1.270 \\
\hline 6 & 0.9915 & 0.9915 & 0.9915 & 0.0000 & 0.9915 & -0.850 \\
\hline
\end{tabular}

Table 2d: Measured absorbed dose and average percentage deviation of solid water phantom against the in-house phantom

\begin{tabular}{|c|c|c|c|c|c|c|}
\hline \multicolumn{7}{|c|}{ TRUNK INHOMOGENEITY $\left(5 \times 5 \mathrm{~cm}^{2}\right.$ FIELD SIZE $)$} \\
\hline FIELDS & \multicolumn{3}{|c|}{ MEASURED ABSORBED DOSE } & STD & AVG & \%DEV \\
\hline 1 & 1.0064 & 1.0041 & 1.0064 & 0.0013 & 1.0056 & 0.560 \\
\hline 2 & 0.9996 & 0.9996 & 0.9996 & 0.0000 & 0.9996 & -0.040 \\
\hline 3 & 0.9859 & 0.9836 & 0.9836 & 0.0013 & 0.9844 & -1.560 \\
\hline 4 & 0.9677 & 0.9699 & 0.9677 & 0.0013 & 0.9684 & -3.160 \\
\hline 5 & 0.9653 & 0.9630 & 0.9630 & 0.0013 & 0.9638 & -1.270 \\
\hline 6 & 0.9765 & 0.9734 & 0.9765 & 0.0018 & 0.9755 & -2.250 \\
\hline
\end{tabular}




\section{Discussion}

A study has been carried out to verify the performance of a Treatment Planning System which uses an Irregular Field Algorithm based on previously published methods $[5,12,13]$. The results were within the range of $\pm 5 \%$ as recommended by ICRU[10] and were consistent with Van Dykwhose variation was within $\pm 4 \%$, [18]. Mijnheer and Brahme were also within 3-3.5\% [4, 15]. The results in table 2(a and b) showed that the Irregular Field Algorithm had a maximum percentage deviation of $-3.39 \%$ and $+2.93 \%$ for six inhomogeneous inserts and bone only inhomogeneous inserts with large field size of $22 \times 24 \mathrm{~cm}^{2}$ respectively. The result for table (2c) for all six inhomogeneous inserts with small field size of $5 \times 5 \mathrm{~cm}^{2}$ had a maximum percentage deviation of $-3.16 \%$. The result for table (2d) showed that the percentage deviation between the solid water phantom and the in-house phantom was $-3.33 \%$, this value further confirms the accuracy of the inhouse phantom. Table (2a) showed an increase in the numerical value for the first five fields, with the highest deviation noticed in the tenth field. The overall percentage deviation range was $0.04 \%$ to $-3.39 \%$. In table (2b), the least percentage deviation was noticed in the sixth field with a value of $-0.85 \%$, with an overall percentage deviation range of $-0.85 \%$ to $+2.93 \%$. In addition, the least deviation were observed for the first field in table (2a) and second field in table (2c) respectively. An overview of this study showed that the designed in-house phantom was within acceptable clinical limits and showed that the in-house phantom can be used for routine clinical test in radiotherapy.

\section{Conclusion}

An in-house phantom has been designed; with hollows containing tissue equivalent materials to mimic the human body using an Elekta-Precise Clinical Linear Accelerator based on Irregular Field Algorithm with an overall variation of $\pm 3 \%$ with the in-house phantom for small and large field sizes. The maximum deviation was recorded in table (2a) with six inhomogeneous inserts with large field size. These values are well within clinical tolerance levels, making this in-house phantom fit for use in radiotherapy department especially those with low budget (African centres) that cannot afford the commercially expensive ones.

\section{References}

[1]. Absorbed Dose Determination in External Beam Radiotherapy, Technical ReportsSeries No. 398, IAEA, Vienna (2000).

[2]. Alam R, Ibbott GS, Pourang R, Nath R. Application of AAPM Radiation Therapy Committee Task Group 23 test package for comparison of two treatment planning systems for photon external beam radiotherapy. Med Phys. 1997, 24:2043-54.

[3]. Animesh Advantages of multiple algorithms support in treatment planning system for external beam dose calculations. $J$ Cancer Res Ther.2005; 1:12-20.

[4]. Brahme A, Chavaudra J, Landberg T, et al. Accuracy requirementsand quality assurance of external beam therapy with photons andelectrons. ActaOncol (Stockholm) 1988; (Suppl. 1):1-76.

[5]. Clarkson JR. Note on Depth Doses in Fields of Irregular Shapes. Brit. J. Radiol. Vol. 14, 1941, pp.265-268.

[6]. Cygler J, Ross J. Electron dose distribution in an anthropomorphic Phantom-verification of Theraplan planning algorithm. Med Dos.1988; 13:155-158.

[7]. Fraass BA. "Quality Assurance for 3-D Treatment Planning.” In Teletherapy: Present and Future. Palta J, Mackie TR (Eds.). Madison: Advanced Medical Publishing. 1996, 253-318.

[8]. Fraass BA, Doppke K, Hunt M, Kutcher G, Starkschall G, Stern R, Van Dyk J. Med. Phys.1998, 25:1773-1829.

[9]. Institute of Physics and Engineering in Medicine (IPEM). Physics aspects of quality control in radiotherapy. IPEM Report 81. York: IPEM, 1999.

[10]. International Commission on Radiation Units and Measurements (ICRU). Dose specifications for reporting external beam therapy with photons and electrons. 1978 ICRU Report 29, Baltimore, MD: ICRU Bethesda, MD.

[11]. Jacky J, White CP. Testing a 3-D radiation therapy planning program. Int. J. RadiatOncolBiolPhys 1990; 18:253-261.

[12]. Johns HE, Cunningham JR. The Physics of Radiology, Third Edition, 1971, pp. 362-363.

[13]. Khan FM. The Physics of Radiation Therapy, Williams and Wilkins, Baltimore, MD., 1984, p.321 and pp.787-794.

[14]. Khan FM.The Physics of Radiation Therapy, Lippincott, Williams and Wilkins,Baltimore, MD (2003).

[15]. Mijnheer BJ, Battermann JJ, Wambersie A. What degree of accuracy is required and can be achieved in photon and neutron therapy? RadiotherOncol.1987; 8: 237-52.

[16]. Podgorsak EB. Radiation Oncology Physics: A handbook for Teachers and Students. Vienna: IAEA publication. 2005.

[17] Shaw JE. (Ed.) “A Guide to Commissioning and Quality Control of Treatment Planning Systems.”The Institution of Physics and Engineering in Medicine and Biology.1994.

[18]. Van Dyk J, Barnett RB, Cygler JE, Shragge PC. Int. J. Radiat. Oncol.Biol. Phys. 1993; 26:261-273.

[19]. Van Dyk J. "Quality Assurance." In Treatment Planning in Radiation Oncology.Khan FM, Potish RA (Eds.). (Baltimore, MD: Williams and Wilkins). 1997, 123-146. 